\title{
Gender differences and psychological factors associated with suicidal ideation among youth in Malaysia
}

This article was published in the following Dove Press journal:

Psychology Research and Behavior Management

28 April 2017

Number of times this article has been viewed

\section{Norhayati lbrahim \\ Noh Amit \\ Normah Che Din \\ Hui Chien Ong}

Health Psychology Program, Faculty of Health Sciences, Universiti Kebangsaan Malaysia, Kuala

Lumpur, Malaysia
Correspondence: Norhayati Ibrahim Health Psychology Program, Faculty of Health Sciences, Universiti Kebangsaan Malaysia, Jalan Raja Muda Abdul Aziz, 50300 Kuala Lumpur, Malaysia

Tel +60 326878174

Fax +60 326878192

Email norhayati70@gmail.com
Abstract: Suicide is a global phenomenon that has been showing an upward trend in recent years. It is the second leading cause of death among youth. Studies on suicidal ideation warrant greater attention, as it leads to suicide attempts and other health risk behaviors. Thus, the objective of this study was to compare gender differences in suicidal ideation and determine the predictors of suicidal ideation among youth. This cross-sectional study was carried out among 232 youths aged between 15 and 25 years from selected urban areas in Malaysia. The results showed that suicidal ideation was higher among male participants compared with female participants. Age was the predictor of suicidal ideation for males, while depression and loss of motivation, as components of hopelessness, were the predictors of suicidal ideation among females. Hence, it is important that professionals conduct early identification tests for suicidality among young people. This will facilitate the early detection of depression and hopelessness, which is important, in order to prevent suicidal behaviors or other problems before these occur. Keywords: suicidal ideation, youth, depression, hopelessness, gender

\section{Introduction}

In recent years, the suicide rate has been increasing and appears to be a serious public health problem in Malaysia. Statistics show that the suicidal rate has increased by $60 \%$ over the past 45 years in Malaysia. It is reported that $>800,000$ people end their life through suicide and $75 \%$ of global suicides occur in low- and middle-income countries. ${ }^{1}$ Past research in Malaysia showed that approximately seven people committed suicide per day, and most of them belonged to the youth category. ${ }^{2}$ Suicidal ideation most likely happens prior to a suicide attempt or suicide, ${ }^{3-6}$ and it involves the tendency to die owing to an inability to cope with life difficulties. ${ }^{7}$ According to Reynolds, ${ }^{8}$ suicidal ideation is a thought, idea, or plan that can lead to harm or/and death. Suicidal ideation becomes severe and worsens when one fails to overcome a problem.

The National Suicide Registry Malaysia had compiled the statistics of suicide cases from 2007 to 2009, and it was found that suicides are committed due to school issues or intimate problems. ${ }^{9}$ According to the National Health and Morbidity Survey conducted by the Ministry of Health Malaysia, ${ }^{10} 6.3 \%$ of the participants reported having suicidal ideation and the youngest age group - 16-19 years old - was most disposed to suicidal thoughts, followed by the 20-24 years age group. The youth category consists of people who are still in their high schools, colleges, universities, and first jobs and are transitioning into adults. There are many reasons that are associated with suicidal ideations among youth, regardless of them being students or working professionals. For example, it could be caused by substance abuse, academic failures, psychological 
disorders, poor social and family relationships, aggression or impulsivity, risky sexual behaviors, and so on. ${ }^{9,11-14}$

One important area of the research is understanding of gender differences in suicidal ideation and suicide behaviors. A previous study showed that males were more likely to die from suicides, while females were more likely to commit suicide attempts. ${ }^{15}$ Besides, female adolescents were more likely to have suicidal thoughts and ideation compared with male adolescents. ${ }^{16,17}$ Regarding the timing of first onset of suicide attempts, Lewinsohn et $\mathrm{al}^{17}$ found that the risk of onset for both boys and girls peaked during mid adolescence. However, young women's risk precipitously dropped after age 18 , whereas young men's risk only slightly decreased in late adolescence.

During the adolescent stage, males and females develop differently with different skills at different times. ${ }^{18}$ There are changes in the actual levels of turbulence once believed to occur at this stage of emotions and concepts. ${ }^{19}$ One of the occasions that distinguishes men and women is dealing with problems in life. Even though both genders have the same goal of solving the problem, they will often go about finding a solution in a very different manner to each other.

Previous research shows that hopelessness is associated with suicide ${ }^{20}$ and is a predictor of suicide. ${ }^{21}$ However, hopelessness could not predict suicide when depression was controlled, which indicates that hopelessness may be linked to suicidal behaviors only under certain circumstances. ${ }^{22}$ Apart from this, depression was found to be a prominent factor in suicidal ideation among youth ${ }^{6,23,24}$ and was mostly diagnosed among adolescents who attempted suicide. ${ }^{23,25,26}$ In past studies, stress, anxiety, and depression positively correlated with suicidal ideation. ${ }^{27-31}$

Suicide is a growing problem among youth today, and it should be prevented as early as possible. Many earlier studies had examined the related psychological and sociodemographic factors and predicted suicidal ideation among youth from different samples. However, studies on youth suicide in Malaysia are still scarce and not many research studies had really focused on gender differences in suicidal ideation. ${ }^{32}$ Studying gender differences among youths with suicidal ideation is important for identifying gender-specific risk factors because the stressors experienced between these two genders also differed. Thus, the planning management and prevention should be based on the gender differences. Hence, the aim of this study was to compare gender differences in suicidal ideation and analyze the factors associated with suicidal ideation and predictors of suicidal ideation based on gender differences.

\section{Methodology \\ Research design}

This cross-sectional study was carried out with youth from selected urban areas in the states of Wilayah Persekutuan and Selangor, Malaysia, from October to December 2014. The urban areas were selected based on the reported number of suicide cases and were located in locality of low to moderate socioeconomic status. ${ }^{1}$ This study was approved by the Universiti Kebangsaan Malaysia Research Ethics Committee (approval number NN-064-2013). The number of participants studied was 232 young people. The inclusion criteria for the selection of the subjects were as follows: aged between 15 and 25 years, able to read and write in Malay or English language, and willing to participate in this study. Written informed consent was obtained from all individual participants included in the study. The purpose of this study was explained, and those who agreed to participate in the study signed a consent form. All the respondents were assured of data confidentiality, and subjects were included on a voluntary basis. Face-to-face interview was conducted at their homes, and upon completion, data were then anonymously keyed into the Statistical Package for the Social Sciences (SPSS) software. The use of face-to-face interview was potential to minimize risk of missing data during the data collection process in the present study.

\section{Instruments}

\section{Suicidal Ideation Scale (SIS)}

The SIS was constructed by Rudd in 1989 to measure the level of severity or tendency of suicidal ideation among young adults. Each item of the SIS is a statement about suicidal ideation from suicidal behavior toward suicide attempts. The scale consists of 10 items, which present critical information about "the presence or absence of suicidal thinking, the intensity of these thoughts, and the presence or absence of prior suicide attempts". ${ }^{33}$

The SIS reported high internal consistency (Cronbach's alpha $=0.86$ ), and overall item-total correlation ranged from 0.45 to $0.74 .{ }^{34}$ Moreover, SIS appeared to have good construct validity as there was a positive correlation between the Centre for Epidemiologic Studies Depression Scale (CES-D) and the Beck Hopelessness Scale (BHS).

\section{I-Item Depression Anxiety Stress Scale (DASS-2 I)}

DASS- $21^{35}$ is a self-administered psychometric test that gauges levels of depression, anxiety, and stress over the previous week. The scale is applicable in both clinical and nonclinical population. There are 21 items in DASS with 
three subscales (depression, anxiety, and stress). Each subscale comprises seven items. The Malay-translated version of DASS-21 was developed by Ramli, Mohd, and Zaini. ${ }^{36}$ The alpha coefficients of DASS-21 ranged from 0.74 to 0.84 . The DASS-21 was further validated through factor analysis, which ranged from 0.39 to 0.73 . The correlation among the scales was between 0.54 and $0.68 .{ }^{36}$

\section{Beck Hopelessness Scale}

The BHS was constructed by Beck et al in 1974 to measure three major aspects of hopelessness: feelings about the future, loss of motivation, and expectations. The test is designed for adults, aged 17-80 years, and consists of 20 items. The psychometric analysis indicated high internal consistency, high reliability, and good concurrent validity of the scale. ${ }^{37}$ The internal reliability coefficients were shown to be reasonably high (Pearson's $r=0.82-0.93$ in seven norm groups), and the BHS test-retest reliability coefficients were found to be modest ( 0.69 after 1 week and 0.66 after 6 weeks) ${ }^{38}$

\section{Data analysis}

The raw data were keyed into the SPSS version 21. Independent $t$-test was carried out to compare suicidal ideation, hopelessness, and psychological distress among males and females. Pearson's correlation was used to test the correlation between the variables, and multiple regression was conducted to examine the role of age, hopelessness, and psychological distress in suicidal ideation among youth.

\section{Results}

The total number of participants was 232. The results in Table 1 show that most participants were aged between 15 and 17 years $(44.8 \%)$, followed by those aged $18-21$ years (27.2\%) and $22-25$ years $(28.0 \%)$. The majority were males (55.6\%), Malays (83.2\%), Muslims (86.6\%), and those who had completed upper secondary education (37.9\%).

The results of independent $t$-test analysis for gender differences based on age, suicidal ideation, hopelessness, and psychological distress indicated that only suicidal ideation, anxiety, loss of motivation, and future expectations showed significant differences in terms of gender. In addition, the findings showed that male participants had a higher mean score than female participants in suicidal ideation, future feeling, loss of motivation, and future expectations, while female participants had a higher mean score than male participants in terms of depression, anxiety, and stress (Table 2).

Pearson's correlations were used to analyze the correlations between suicidal ideation and age, hopelessness, depression, anxiety, and stress for males and females. The overall
Table I Demographic characteristics of the respondents

\begin{tabular}{lll}
\hline Characteristics & $\mathbf{n}$ & $\%$ \\
\hline Age (years) & 104 & 44.8 \\
I5-I7 & 63 & 27.2 \\
I8-2I & 65 & 28.0 \\
$22-25$ & & \\
Gender & 129 & 55.6 \\
Male & 103 & 44.4 \\
Female & & \\
Ethnicity & 193 & 83.2 \\
Malays & 41 & 16.9 \\
Non-Malays & & \\
Religion & 201 & 86.6 \\
Islam & 31 & 13.3 \\
Other religions & & \\
Highest education & 4 & 1.7 \\
No schooling & 67 & 28.9 \\
Primary education & 88 & 37.9 \\
Secondary education & 73 & 31.5 \\
Tertiary education &
\end{tabular}

Table 2 Gender differences in suicidal ideation, depression, stress, anxiety, and hopelessness

\begin{tabular}{llllll}
\hline Variables & Gender & $\mathbf{n}$ & Mean & SD & $\boldsymbol{t}$ \\
\hline Suicidal ideation & Male & 129 & 14.89 & 7.920 & $2.049^{*}$ \\
& Female & 103 & 13.01 & 6.063 & \\
Depression & Male & 129 & 4.87 & 2.887 & -1.898 \\
& Female & 103 & 5.71 & 3.680 & \\
Anxiety & Male & 129 & 5.67 & 3.058 & $-2.056^{*}$ \\
& Female & 103 & 6.59 & 3.661 & \\
Stress & Male & 129 & 6.84 & 3.069 & -1.919 \\
& Female & 103 & 7.74 & 3.896 & \\
Future feeling & Male & 129 & 4.60 & 1.296 & 0.767 \\
& Female & 103 & 4.48 & 1.056 & \\
Loss of motivation & Male & 129 & 3.53 & 2.934 & $3.526 * *$ \\
& Female & 103 & 2.40 & 1.957 & \\
Future expectations & Male & 129 & 2.79 & 1.604 & $1.977^{*}$ \\
& Female & 103 & 2.41 & 1.271 & \\
\hline
\end{tabular}

Notes: $* p<0.05 ; * * p<0.01$.

Abbreviation: SD, standard deviation.

results showed that age was negatively correlated with suicidal ideation $(r=-0.275)$. All components in hopelessness (future feeling, loss of motivation, and future expectations) were significantly correlated with suicidal ideation. This finding showed that future feeling was negatively correlated with suicidal ideation $(r=-0.147)$, while loss of motivation $(r=0.235)$ and future expectations $(r=0.210)$ were positively correlated with suicidal ideation. In addition, it showed that depression $(r=0.281)$ and anxiety $(r=0.171)$ were positively correlated with suicidal ideation.

The results in Table 3 are divided for males and females. It shows that in males, age is negatively correlated with suicidal ideation $(r=-0.334)$, while depression is positively correlated with suicidal ideation $(r=0.184)$. On the other hand, 
Table 3 Correlations of age, hopelessness, and depression with suicidal ideation

\begin{tabular}{llll}
\hline Variables & Component & Suicidal ideation (male) & Suicidal ideation (female) \\
\hline Age & Not applicable & $-0.334^{*}$ & -0.161 \\
Hopelessness & Future feeling & -0.131 & $-0.204^{* *}$ \\
& Loss of motivation & 0.143 & $0.386^{*}$ \\
& Future expectations & 0.168 & $0.255^{* *}$ \\
Depression, anxiety, and stress & Depression & $0.184^{* *}$ & $0.477^{*}$ \\
& Anxiety & 0.129 & $0.292^{*}$ \\
& Stress & 0.045 & $0.215^{*}$ \\
\hline
\end{tabular}

Notes: $* p<0.05 ; * p<0.01$.

hopelessness components (future feeling, loss of motivation, and future expectations), depression, anxiety, and stress were significantly correlated with suicidal ideation among females.

Furthermore, the results using multiple regression showed that age is the only predictor of suicidal ideation among males, explaining $15.4 \%$ of the variance in suicidal ideation. On the other hand, depression was the best predictor of suicidal ideation among females, followed by loss of motivation, explaining $34 \%$ of the variance in suicidal ideation.

\section{Discussion}

In earlier research studies, findings showed that females reported a higher tendency for suicidal thoughts and ideation compared to male adolescents, ${ }^{16,17}$ which opposes the findings of this study. However, the latest findings in Malaysia showed that the level of suicidal ideation among male students is higher compared to female students, ${ }^{32}$ which is in agreement with this study. This suggested that the males in this study might be more vulnerable to suicidal ideation, probably due to conduct problems as these seemed to increase the risk of suicidal thoughts, as found in other research works. ${ }^{12}$

The reasons of higher suicidal ideation among men could be attributed to a few factors. 1) In dealing with personal and also work-related stress, men are less likely to share and discuss about their problems or difficulty with their significant people and attending to psychological services compared to women..$^{39-41}$ 2) There are gender differences in terms of expression of emotion across cultures. In some Asian culture, men are expected to be reserved in terms of expression of sad emotion compared to women. This reflects to masculine identity of men in Asian cultures where they are expected to be strong not only physically but also emotionally. ${ }^{42}$ Therefore, there is a tendency among small number of young people to have suicide thinking when they are under stress. In this stressful situation, they can be overwhelmed by irrational thought, negative emotion, feeling of hopelessness, and lack of social support, problem solving, and coping skills in dealing with their stressful life events. ${ }^{39,41}$
In addition, the findings of this study also showed that age is a predictor of suicidal ideation and is negatively correlated with suicidal ideation. This suggests that the younger they are, the more likely they will have suicidal ideation. It is probably due to depression and other life adversities, which later lead to a suicide. ${ }^{43}$ However, Fergusson et al ${ }^{44}$ estimated that the lifetime prevalence of suicidal ideation in a large birth cohort of boys climbed from a modest $9.5 \%$ at age 16 years to $24.5 \%$ at age 21 years. Peak levels of past-year ideation and plans occurred during mid adolescence for girls, but slowly increased through late adolescence for boys. ${ }^{45}$ This can also be attributed to their limited coping skills and experience in life and also the way they solve the problem. Usually, women try to solve a problem by relying on the help of those close to them. They talk about their problem, discussing the situation in detail and how they could solve it. On the other hand, men approach problem solving with much less communication, and thus they keep their problem that may lead to suicidal ideation. This may reflect gender differences in the developmental maturity; women normally mature early compared to men. This may indirectly affect the differences in terms of coping skills and problem solving between men and women. ${ }^{18,46}$

Even though in this study males reported higher suicidal ideation compared to females, females suffered from depression at a much higher rate than males, which might show that they are at a higher risk for suicide. However, based on a previous finding, ${ }^{47}$ females are less likely to commit suicide compared to males. This can be attributed to the ways in which females deal with problems and interact with others. They tend to share their experiences with friends, discuss their feelings, seek feedback, and take advice. ${ }^{48}$ This study also found that females showed higher anxiety and stress than males. This is in line with a past finding that reported that female Vietnamese secondary school students had higher depression and anxiety compared to males. ${ }^{14}$ Although it seemed that females had a higher rate of suicidal thoughts, males showed more 
tendency to commit suicide due to fearlessness of death and physical pain insensitivity. ${ }^{43,49,50}$ The number of cases of suicidal deaths was actually much higher (four times) among males than among females. ${ }^{51}$

The findings of this study showed that hopelessness was significantly correlated with suicidal ideation, similar to other studies. ${ }^{13,52}$ In this study, future feelings of hopelessness was negatively correlated with suicidal ideation, while loss of motivation and future expectations were positively correlated with suicidal ideation among female youth in Malaysia. A previous finding had also found that hopelessness is one of the strongest predictors of adolescent suicidal ideation in Hong Kong. ${ }^{13}$ According to Rutter and Behrendt, ${ }^{53}$ hopelessness can predict suicidal tendency among adolescents. Individuals with high levels of hopelessness may isolate themselves and exhibit a less help-seeking behavior, ${ }^{52}$ and hopelessness is also considered as a significant risk factor for suicide. ${ }^{52,54}$

Depression and anxiety are some of the mental health issues that affect adolescents' lives and can lead to family conflicts, substance abuse, social issues, violence, and suicide. ${ }^{14}$ The findings in this study showed that there is an increase in suicidal ideation among youth nowadays and suicidal ideation is correlated positively with depression in both males and females. Moreover, this study found that depression was the strongest predictor of suicidal ideation. Earlier studies had also found that depression was the leading factor in suicidal ideation among youth, ${ }^{6,23,24}$ as it was found that the majority of the adolescents who attempted suicide met the diagnostic criteria for depression. ${ }^{23,25,26}$ In addition, suicidal thoughts increased their risks of psychiatric problems, suicide attempt, and suicide ${ }^{55-57}$ However, Lamis and Lester $^{58}$ found that depression is only a risk factor among female undergraduates. Another research found increasing rate of depressive symptomatology among females compared to males during early adolescence. ${ }^{59,60} \mathrm{~A}$ previous study found that $11 \%$ of youth were inflicted by depressive disorder by 18 years of age, which was caused by possible risk factors such as substance abuse, issues involving self-esteem, relationship problems, family history, psychological issues, and psychological conditions. ${ }^{61}$ Although depression was associated with suicidal ideation, Pinto and Whisman ${ }^{62}$ suggested that depressive symptomatology alone was not sufficient to predict the risk for suicide. Based on this study, depressive symptoms combined with hopelessness tend to increase the risk of suicide, particularly among female youth.

The findings of this study also revealed that suicidal ideation was positively correlated with anxiety, similar to the results of several past research on anxiety disorders. ${ }^{30,63,64}$
Greene et $\mathrm{al}^{65}$ found that generalized anxiety symptoms were associated with suicidality in children and adolescents who were referred for mental health assessments. Another previous study claimed depression and anxiety to be strong predictors of suicidal ideation among youth. ${ }^{66}$ It can be influenced by many factors such as high educational stress, lower education and income of parents, and emotional abuse in the family. ${ }^{14}$ Stress is also an important factor associated with suicidal ideation, and past studies suggest that stress may be due to academic pressure, family issues, ${ }^{14}$ or sexual behaviors and other unhealthy risk behaviors. ${ }^{67}$ Similar to any youth population of developing countries, Malaysian youth experience modernization and are affected by globalization. This leads to the needs of the family to improve their socioeconomic status. Therefore, educational achievement is the best platform for the Malaysian youth to secure a job in order to improve the socioeconomic condition of their family. The common expectation of parents from their children is to succeed in education and secure a job. ${ }^{68,69}$ This expectation is indirectly potential to promote stress among Malaysian youth. ${ }^{70}$ At this stage of development, peer influence is also strong. Therefore, in dealing with stress, Malaysian youth will easily consult with their peer compared to their parents. This may affect their decision and action in problem solving and coping with their stress. Besides this, in Malaysian culture, youth are expected to adhere to the gender role and identity. In terms of expression of emotion between genders, men are expected to be physically and emotionally strong. This is an important reflection of the masculine identity of Malaysian youth. ${ }^{42}$

However, in this study, anxiety and stress were not found to be predictors of suicidal ideation, which is in line with the findings by Ibrahim et $\mathrm{al}^{27}$ on suicidal ideation among adolescents in Malaysia. Anxiety and stress symptoms might not be strong indicators of suicidal ideation, as they only explain the outcome of worries and stress and might not lead to suicidal behavior.

\section{Limitations}

This study has a few limitations. With regard to research design, the present study applied cross-sectional design; hence, there is no causal relationship established. All measures were taken at one point in time, which means it was impossible to determine the time order of the variables. Our findings are limited to youth population in the urban community. Therefore, the results cannot be generalized to be included in other populations, especially in the rural community. This study just focused on the effect of depression and hopelessness on 
suicidal ideation; hence, multiple risk factors should be added to the actual study to assess suicidal ideation. Perhaps, future research can examine the risk factors and protective factors simultaneously, which would help us identify the factors that are strong predictors of suicidal ideation.

\section{Conclusion}

The results from this study provide empirical support to the findings that age, hopelessness, depression, anxiety, and stress are correlated with suicidal ideation and some of the variables become predictors. Hence, strategies and intervention programs should be implemented to strengthen individuals in managing distress and suicidal ideation. Depression and hopelessness, especially loss of motivation, are important factors and should be given due attention in any program related to youth in dealing with circumstances that lead to suicidal behavior, considering that the suicide rate is increasing day by day. If there is no early intervention program, especially on suicidal ideation, it will cause a bad impact and a loss to the country because youth are leaders of tomorrow and an empowered young generation is a pillar of nation's future.

\section{Acknowledgments}

The authors thank all the participants who were involved in this study. This study was funded by grant code number: NN-2014002 from the Prime Minister's Department, Malaysia.

\section{Disclosure}

The authors report no conflicts of interest in this work.

\section{References}

1. World Health Organization [WHO]. Suicide data; 2015. Available from: http://www.who.int/mental_health/prevention/suicide/suicideprevent/ en/. Accessed October 19, 2016.

2. Aishvarya S, Maniam T, Sidi H, Oei TPS. Suicide ideation and intent in Malaysia: a review of the literature. Compr Psychiatry. 2014;55(suppl 1): S95-S100.

3. Brent DA, Johnson B, Bartle S, et al. Personality disorder, tendency to impulsive violence, and suicidal behavior in adolescents. $J$ Am Acad Child Adolesc Psychiatry. 1993;32(1):69-75.

4. Gili-Planas M, Roca-Bennasar M, Ferrer-Perez V, Bernardo-Arroyo M. Suicidal ideation, psychiatric disorder, and medical illness in a community epidemiological study. Suicide Life Threat Behav. 2001;31(2):207-213.

5. Lewinsohn PM, Rohde P, Seeley JR. Adolescent suicidal ideation and attempts: prevalence, risk factors, and clinical implications. Clin Psychol Sci Pract. 1996;3(1):25-46.

6. Reinherz HZ, Giaconia RM, Silverman AB, et al. Early psychosocial risks for adolescent suicidal ideation and attempts. J Am Acad Child Adolesc Psychiatry. 1995;34(5):599-611.

7. Mind. Suicidal feelings; 2016. Available from: http://www.mind.org. uk/information-support/types-of-mental-health-problems/suicidalfeelings/\#.V_xoqfl97IV. Accessed October 19, 2016.
8. Reynolds W. Suicidal Ideation Questionnaire: Professional Manual. Odessa, FL: Psychological Assessment Resources. Inc; 1988.

9. Khan A, Mustaffa MS, Hamdan AR, Ahmad R. Influence of psychological factors on suicide ideation among Malaysian and Indian adolescent. Procedia Soc Behav Sci. 2014;143:347-351.

10. Institute for Public Health. National Health and Morbidity Survey 2011. Kuala Lumpur: Ministry of Health Malaysia; 2011.

11. Arria AM, O'Grady KE, Caldeira KM, Vincent KB, Wilcox HC, Wish ED. Suicide ideation among college students: a multivariate analysis. Arch Suicide Res. 2009;13(3):230-246.

12. Strandheim A, Bjerkeset O, Gunnell D, Bjørnelv S, Holmen TL, Bentzen N. Risk factors for suicidal thoughts in adolescence-a prospective cohort study: the Young-HUNT study. BMJ Open. 2014; 4(8): 005867.

13. Kwok S, Shek D. Hopelessness, parent-adolescent communication, and suicidal ideation among Chinese adolescents in Hong Kong. Suicide Life Threat Behav. 2010;40(3):224-233.

14. Nguyen DT, Dedding C, Pham TT, Wright P, Bunders J. Depression, anxiety, and suicidal ideation among Vietnamese secondary school students and proposed solutions: a cross-sectional study. BMC Public Health. 2013;13(1): 1.

15. Befrienders Worldwide [webpage on the Internet]. Suicide statistics; 2016. Available from: http://www.befrienders.org/suicide-statistics. Accessed October 5, 2016.

16. Grøholt B, Ekeberg Ø, Wichstrøm L, Haldorsen T. Sex differences in adolescent suicides in Norway, 1990-1992. Suicide Life Threat Behav. 1999;29(4):295-308.

17. Lewinsohn PM, Rohde P, Seeley JR, Baldwin CL. Gender differences in suicide attempts from adolescence to young adulthood. $J$ Am Acad Child Adolesc Psychiatry. 2001;40(4):427-434.

18. Berk LE. Development through the Lifespan. 5th ed. Upper Saddle River, NJ: Pearson Education; 2010.

19. Perry DG, Pauletti RE. Gender and adolescent development. $J$ Res Adolesc. 2011;21(1):61-74.

20. Wetzel R, Margulies T, Davis R, Karam E. Hopelessness, depression, and suicide intent. J Clin Psychiatry. 1980;41(5):159-160.

21. Beck AT, Brown G, Steer RA. Prediction of eventual suicide in psychiatric inpatients by clinical ratings of hopelessness. J Consult Clin Psychol. 1989;57(2):309.

22. Dori GA, Overholser JC. Depression, hopelessness, and self-esteem: accounting for suicidality in adolescent psychiatric inpatients. Suicide Life Threat Behav. 1999;29(4):309-318.

23. Gould MS, King R, Greenwald S, et al. Psychopathology associated with suicidal ideation and attempts among children and adolescents. J Am Acad Child Adolesc Psychiatry. 1998;37(9):915-923.

24. Galaif ER, Sussman S, Newcomb MD, Locke TF. Suicidality, depression, and alcohol use among adolescents: a review of empirical findings. Int J Adolesc Med Health. 2007;19(1):27.

25. Beautrais AL, Joyce PR, Mulder RT. Youth suicide attempts: a social and demographic profile. Aust NZJ Psychiatry. 1998;32(3):349-357.

26. Goldston DB, Daniel SS, Reboussin BA, Reboussin DM, Kelley AE, Frazier PH. Psychiatric diagnoses of previous suicide attempters, firsttime attempters, and repeat attempters on an adolescent inpatient psychiatry unit. JAm Acad Child Adolesc Psychiatry. 1998;37(9):924-932.

27. Ibrahim N, Amit N, Suen MWY. Psychological factors as predictors of suicidal ideation among adolescents in Malaysia. PLoS One. 2014;9(10):e110670.

28. Singh R, Joshi HL. Suicidal ideation in relation to depression, life stress and personality among college students. J Indian Acad Appl Psychol. 2008;34(2):259-265.

29. Takeuchi T, Nakao M. The relationship between suicidal ideation and symptoms of depression in Japanese workers: a cross-sectional study. BMJ Open. 2013;3(11):e003643.

30. Weissman MM, Klerman GL, Markowitz JS, Ouellette R. Suicidal ideation and suicide attempts in panic disorder and attacks. $N$ Engl $J$ Med. 1989;321(18):1209-1214. 
31. Gould MS, Greenberg T, Velting DM, Shaffer D. Youth suicide risk and preventive interventions: a review of the past 10 years. J Am Acad Child Adolesc Psychiatry. 2003;42(4):386-405.

32. Mustaffa S, Aziz R, Mahmood MN, Shuib S. Depression and suicidal ideation among university students. Procedia Soc Behav Sci. 2014;116:4205-4208.

33. Luxton DD, Rudd MD, Reger MA, Gahm GA. A psychometric study of the suicide ideation scale. Arch Suicide Res. 2011;15(3):250-258.

34. Rudd MD. The prevalence of suicidal ideation among college students. Suicide Life Threat Behav. 1989;19(2):173-183.

35. Antony MM, Bieling PJ, Cox BJ, Enns MW, Swinson RP. Psychometric properties of the 42-item and 21-item versions of the Depression Anxiety Stress Scales in clinical groups and a community sample. Psychol Assess. 1998;10(2):176.

36. Musa R, Fadzil MA, Zain Z. Translation, validation and psychometric properties of Bahasa Malaysia version of the Depression Anxiety and Stress Scales (DASS). ASEAN J Psychiatry. 2007;8(2):82-89.

37. Beck AT, Weissman A, Lester D, Trexler L. The measurement of pessimism: the hopelessness scale. J Consult Clin Psychol. 1974;42(6):861

38. Aiken LR. Psychological Testing and Assessment. New York, NY: Allyn \& Bacon; 2002.

39. Charbonneau AM, Mezulis AH, Hyde JS. Stress and emotional reactivity as explanations for gender differences in adolescents' depressive symptoms. J Youth Adolesc. 2009;38(8):1050-1058

40. Galdas PM, Cheater F, Marshall P. Men and health help-seeking behaviour: literature review. $J$ Adv Nurs. 2005;49(6):616-623.

41. Verma R, Balhara YPS, Gupta CS. Gender differences in stress response: role of developmental and biological determinants. Ind Psychiatry J. 2011;20(1):4.

42. Naghavi F, Redzuan M. The relationship between gender and emotional intelligence. World Appl Sci J. 2011;15(4):555-561.

43. Beaton SJ, Forster P. Insights into men's suicide. InPsych. 2012;34(4):16-17.

44. Fergusson DM, Woodward LJ, Horwood LJ. Risk factors and life processes associated with the onset of suicidal behaviour during adolescence and early adulthood. Psychol Med. 2000;30(1):23-39.

45. Boeninger DK, Masyn KE, Feldman BJ, Conger RD. Sex differences in developmental trends of suicide ideation, plans, and attempts among European American adolescents. Suicide Life Threat Behav. 2010;40(5):451-464.

46. Matud MP. Gender differences in stress and coping styles. Pers Individ Dif. 2004;37(7):1401-1415.

47. Abram KM, Choe JY, Washburn JJ, Teplin LA, King DC, Dulcan MK Suicidal ideation and behaviors among youths in juvenile detention J Am Acad Child Adolesc Psychiatry. 2008;47(3):291-300.

48. Murphy GE. Why women are less likely than men to commit suicide. Compr Psychiatry. 1998;39(4):165-175.

49. Joiner T. Lonely at the Top: The High Cost of Men's Success. New York, NY: Palgrave Macmillan; 2011.

50. Witte TK, Gordon KH, Smith PN, Van Orden KA. Stoicism and sensation seeking: male vulnerabilities for the acquired capability for suicide. J Res Pers. 2012;46(4):384-392.

51. Centers for Disease Control and Prevention [CDC]. Suicide Datasheet; 2015. Available from: http://www.cdc.gov/ViolencePrevention/pdf/ suicide-Datasheet-a.pdf. Accessed October 5, 2016.

52. Forintos DP, Sallai J, Rózsa S. Adaptation of the Beck Hopelessness Scale in Hungary. Psychol Topics. 2010;19(2):307-321.
53. Rutter PA, Behrendt AE. Adolescent suicide risk: four psychosocial factors. Adolescence. 2004;39(154):295.

54. Glanz LM, Haas GL, Sweeney JA. Assessment of hopelessness in suicidal patients. Clin Psychol Rev. 1995;15(1):49-64.

55. Reinherz HZ, Tanner JL, Berger SR, Beardslee WR, Fitzmaurice GM. Adolescent suicidal ideation as predictive of psychopathology, suicidal behavior, and compromised functioning at age 30. Am J Psychiatry. 2006;163(7):1226-1232.

56. Herba C, Ferdinand R, van der Ende J, Verhulst F. Long-term associations of childhood suicide ideation. JAm Acad Child Adolesc Psychiatry. 2007;46(11):1473

57. Kerr DC, Owen LD, Pears KC, Capaldi DM. Prevalence of suicidal ideation among boys and men assessed annually from ages 9 to 29 years. Suicide Life Threat Behav. 2008;38(4):390-402.

58. Lamis DA, Lester D. Gender differences in risk and protective factors for suicidal ideation among college students. J College Stud Psychother. 2013;27(1):62-77.

59. Ge X, Lorenz FO, Conger RD, Elder GH, Simons RL. Trajectories of stressful life events and depressive symptoms during adolescence. Dev Psychol. 1994;30(4):467.

60. Ge X, Natsuaki MN, Conger RD. Trajectories of depressive symptoms and stressful life events among male and female adolescents in divorced and nondivorced families. Dev Psychopathol. 2006;18(01):253-273.

61. Wyatt LC, Ung T, Park R, Kwon SC, Trinh-Shevrin C. Risk factors of suicide and depression among Asian American, Native Hawaiian, and Pacific Islander youth: a systematic literature review. $J$ Health Care Poor Underserved. 2015;26(2):191.

62. Pinto A, Whisman MA. Negative affect and cognitive biases in suicidal and nonsuicidal hospitalized adolescents. J Am Acad Child Adolesc Psychiatry. 1996;35(2):158-165.

63. Sareen J, Cox BJ, Afifi TO, et al. Anxiety disorders and risk for suicidal ideation and suicide attempts: a population-based longitudinal study of adults. Arch Gen Psychiatry. 2005;62(11):1249-1257.

64. Nepon J, Belik SL, Bolton J, Sareen J. The relationship between anxiety disorders and suicide attempts: findings from the National Epidemiologic Survey on Alcohol and Related Conditions. Depress Anxiety. 2010;27(9):791-798.

65. Greene FN, Chorpita BF, Austin AA. Examining youth anxiety symptoms and suicidal ideation in the context of the tripartite model of emotion. J Psychopathol Behav Assess. 2009;31(4):405-411.

66. Izadinia N, Amiri M, Jahromi R, Hamidi S. A study of relationship between suicidal ideas, depression, anxiety, resiliency, daily stresses and mental health among Tehran university students. Procedia Soc Behav Sci. 2010;5:1615-1619.

67. Peltzer K, Pengpid S. Suicidal ideation and associated factors among school-going adolescents in Thailand. Int J Environ Res Public Health. 2012;9(2):462-473.

68. Fucci S, Cavaletto GM. Educational choices of the elites. Autonomy and responsibility of Italian and foreign children between family and school. Procedia Soc Behav Sci. 2012;46:4990-4994.

69. Wang M-T, Degol J. Motivational pathways to STEM career choices: using expectancy-value perspective to understand individual and gender differences in STEM fields. Dev Rev. 2013;33(4):304-340.

70. Elias H, Ping WS, Abdullah MC. Stress and academic achievement among undergraduate students in Universiti Putra Malaysia. Procedia Soc Behav Sci. 2011;29:646-655.
Psychology Research and Behavior Management

\section{Publish your work in this journal}

Psychology Research and Behavior Management is an international, peerreviewed, open access journal focusing on the science of psychology and its application in behavior management to develop improved outcomes in the clinical, educational, sports and business arenas. Specific topics covered in the journal include: Neuroscience, memory and decision making; Behavior modification and management; Clinical applications; Business and sports performance management; Social and developmental studies; Animal studies. The manuscript management system is completely online and includes a very quick and fair peer-review system, which is all easy to use. Visit http://www. dovepress.com/testimonials.php to read real quotes from published authors. 